\title{
Android based Portable Health Support System
}

\author{
K. Nikita \\ CSE GITAM \\ Bangalore, India
}

\author{
A. Sai Trilok \\ CSE GITAM \\ Bangalore, India
}

\author{
B. L. Narasimha Rao \\ CSE GITAM \\ Bangalore, India
}

\author{
Eikshitha.Y \\ CSE GITAM \\ Bangalore, India
}

\author{
Mylara Reddy C \\ CSE GITAM \\ Bangalore, India
}

\begin{abstract}
Large number of people are becoming patients of variety of diseases due to various reasons. World is facing the problem of death of people due to the heart attacks. In order to avoid such kind of problems we present a work (a device that consists of various sensors) that help minimize the number of deaths. Sensors continuously read parameters such as temperature and heart beat rate of a person. Data from sensors are transferred to a smart electronic gadget through GSM module. Proposed system helps patients to walk freely and their movement can be observed whenever required. Electronic gadget with an installed application can detect the possibility of heart attack using the received data. When any abnormalities are found a message that consists of patient's health condition and exact location of patient is sent to doctor. This information helps in providing urgent medical service to patient.
\end{abstract}

Keywords - Internet of Things, Pulse sensor, Body temp sensor, BP sensor, GSM.

\section{INTRODUCTION}

Gathering and transfer of health condition of people in real time manner is crucial and critical for rapid medical assistance and thereby increase the chances of saving a life. Smart hardware and communication technology, and Internet of Things (IoT) play vital role in reducing death rate. IoT involves thousands of hundreds of interconnected devices, which interact with each other, sense, share information through various communication technologies such as $\mathrm{WiFi}$, Bluetooth, etc. Technology advancement in physiological sensing devices and wireless communication provided by the IoT has brought paradigm shift in health monitoring on a real time basis.

As we all know in-patients of hospitals are periodically monitored by doctors. In spite of periodic monitoring sometimes patients die may be due to unavailability timely treatment or doctors presence at the right time, or lengthy periodic intervals. To overcome this problem, hospitals must have to employ large number of medical staff resulting in more expenditure due to payrolls, maintenance, etc. Though there exists sufficient number of medical staff it's not worth to dedicate a doctor and/or nurse on full time to continuously observe each and every patient biological parameters unless the patient is in very critical condition. For continuous observation of heart beat, temperature and blood pressure of a patient he/she need to be in hospital. It results in hospital expenditure that is extremely difficult to bear by poor and middle class families. To overcome this problem the proposed system helps patient to stay at home. Patient's data is continuously monitored and data transfer interval can also be set ( 2 or 3 minutes). Doctor can view the health status of the patient regularly using mobile android application (figure 17).

Proposed system presented in this article is a smart health monitoring system use sensors to capture patient's health condition and predicts patient's health condition. Collected data is sent to doctors mobile devices and thereby can access health information from anywhere and at any time using android application. In particular this system helps in providing medical assistance to patients effectively.

This system aims at providing cost effective medical assistance to the patients at right time by sending the rescue team to the location immediately without any delay.

\section{RELATED WORK}

To address the issues of data storage, synchronization of health care systems with health care institutions a mobile application was designed and developed [1]. The proposed system did not address the problem of monitoring patients and alert mechanism. A user friendly smart watch that sense patient physiological conditions was proposed in [2]. A geolocation-based heart rate monitoring system [3] that monitors heart rate was proposed. A mobile application to trigger alarm messages was proposed. An approach that is a combination of genetic research and environmental monitoring in real time by using an integrated system of information technology and IOT [4] was proposed to assist smart cities.

Smart health care system [5] using IoT was proposed to address the shortage of health care professionals at remote villages. Doctors monitor patients from remote places/villages. Proposed system makes use of parameters such as Electrocardiograph (ECG), body temperature, blood pressure (BP), heart beat rate, glucose level detection and galvanic skin response of a patient. Smart phone based applications [6] that help to monitor a person biological data like body mass index (BMI), number of steps walked in a day, heart beat was proposed. Proposed system makes use of fuzzy and first-order logic to perform the task. Smart watch based health monitoring system [7] that capture and monitoring various biological parameters of a patient was proposed. 
Captured data are stored in a cloud for further processing and alert patient family members and nearby medical staff. To assist doctors monitor their patients treated for cardiac arrest particularly undergone surgery and voluntarily fail to attend regular check-ups an IOT augmented health monitoring system was implemented by A. N. Reddy et al. [8]. Noninvasive smart health monitoring system using optical fiber interferometers [9] that collect breath and heart beat data. Most of the proposed systems are either costlier, remote geographical locations.

Monitoring of patients and the environment is known to be an individual system in health care. Yet there is no convergence of both. The medical practitioner must be in the health center to track the patient and produce a report. There was no other alternative. Occasionally, if the medical practitioner is not present in the health center, he or she will give the patient's report via a fax or a call that is very risky. Therefore, a smartphone application is mandatory for the medical practitioner so that he can deliver a prescription from anywhere.

In this work we implement an Android based portable health support system. In our system the sensors read blood pressure and temperature continuously and display the values in android application. The doctor can open the application and check the values displayed by the sensors. Not only viewing the values he/she can also prescribe the medicine to the patient whenever required. If the values of the sensors exceed the threshold value then abnormal condition is detected. If an abnormal condition is detected, a message is immediately sent to the medical practitioner as well as to the hospital. The message includes the condition of the patient to understand the emergency situation. Objectives of this work are:

- Design and develop hardware system that capture blood pressure, heart beat rate and transfer the same to processing services

- Design and develop android based mobile application that help doctors to view patients health condition and also prescribe medicine

\section{SYSTEM MODEL}

Proposed system given in fig. 1 consists of various hardware components such as Arduino UNO microcontroller, sensors to sense different biological parameters, GPS and GSM

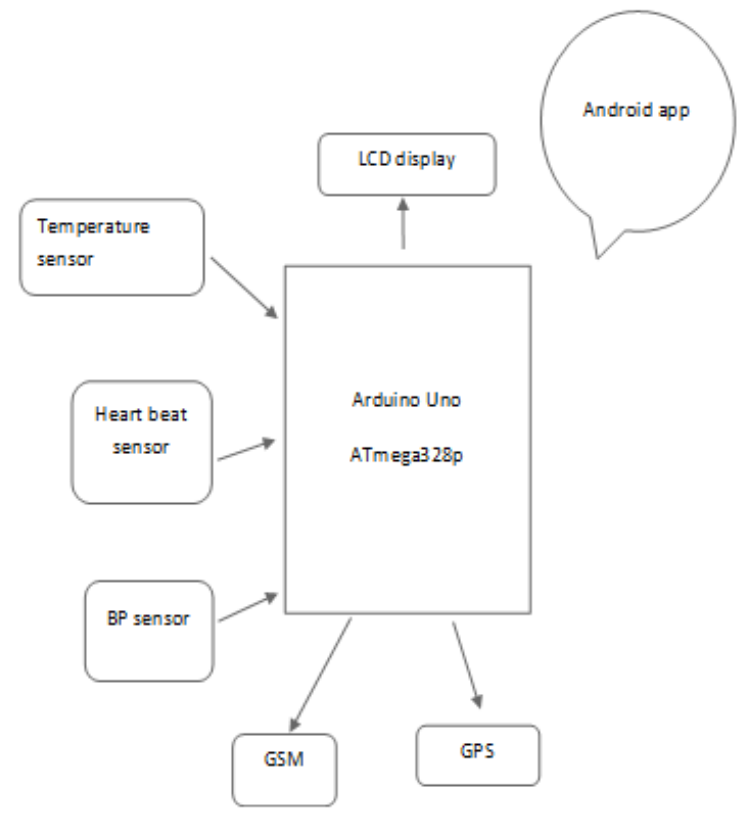

Figure 1. Architecture of the proposed system.

\section{A. Arduino UNO}

Arduino is a prototyping open source electronics framework focused on software and hardware that are scalable. Arduino, given in figure 2, can sense the world through a number of sensors, and can monitor lighting, motors and other actuators in its surroundings. The board's microcontroller is controlled by Arduino and Arduino Creation System. Arduino projects may either be autonomous or interact with computer-based applications. This is inexpensive, cross platform and open source. The Arduino is focused on the microcontrollers Atmel's ATMEGA8 and ATMEGA168.

\section{Technical Specifications:}

Micro-controller: microchipATmega328 P

Operational Voltage: 5 Volts

Inlet Voltage: 7 to $20 \mathrm{~V}$

Analog I / O pins: 14 (plus PWM output 6)

SRAM: $2 \mathrm{~KB}$

EEPROM: $1 \mathrm{~KB}$

Clock Speed: $16 \mathrm{MHz}$

Flash Memory: $45 \mathrm{kB}$, plus $2 \mathrm{~Kb}$ of SRAM (EPROM: $1 \mathrm{~KB}$ )

Length: $68.6 \mathrm{~mm}$

Width: $53.4 \mathrm{~mm}$

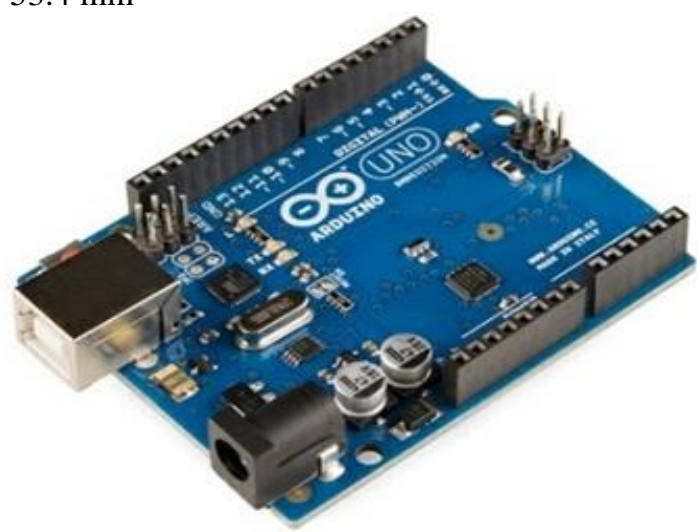

Figure 2. Arduino UNO 


\section{B. Heart Pulse Sensor (SEN-11574)}

The Pulse Monitor is an integrated Arduino heart rate plugand-play device given in figure 3. Students, designers, athletes and software developers who wish to conveniently use live heart rate data in their apps can make use of it. It is also fitted with an open source tracking software that pulse graphs in real time. This includes a light Lead, noise and 3 pins (Gnd, Vcc, Analog A0).

\section{Working:}

A light emission diode and a sensor like a light resistor or photodiode is part of a basic heartbeat system. The waves of the heartbeat produce a difference in blood pressure in multiple areas of the body. The light source, that is, the light produced by the lead, represents a (finger-tissue) or transmits a light (earlobe). When a tissues are lit with a light source. The blood removes some of the light and the light emitted or mirrored by the light detector is provided. The absorption of light relies on the amount of blood in the tissue. The detector output is electric and proportional to the heart function. In addition, this signal is a DC signal that is associated with heart beat and triggered by pulsative shifts in arterial blood pressure that is superimposed on the DC signal by the AC part in relation to tissue that blood volume. The key necessity is therefore to isolate this AC portion as it is necessary.

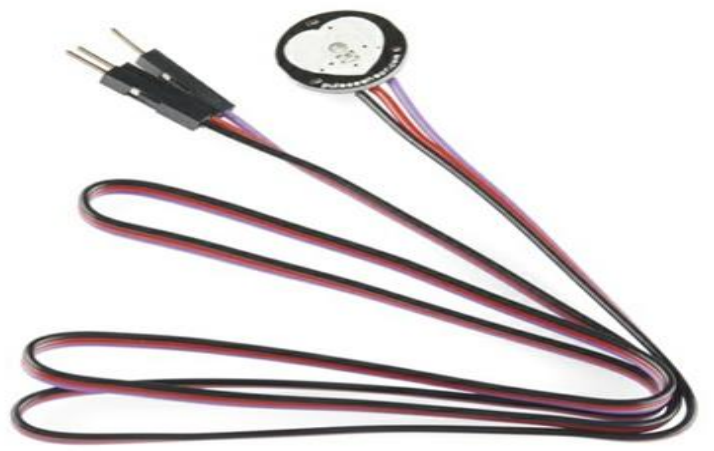

Figure 3. Heart Pulse Sensor

\section{Temperature Sensor (LM-35)}

LM35, given in figure 4, is analog sensor with a degree centigrade proportional electrical efficiency. To have a standard sensitivity, LM35 sensor needs no external adjustment or cutting. LM35 is a temperature control device with a temperature dependent analog output voltage. The LM35 frequency is $10 \mathrm{mV} /$ degree Celsius. This is provided by Celsius voltage. The output voltage often decreases with rising temperature. This comprises 3 pins (4 to $20 \mathrm{v}$, out, Gnd).

The temperature can be compared to a thermistor more precisely. This sensor emits high voltage than thermocouples and doesn't have to boost the output voltage. The LM35 has a voltage equal to the temperature of the Celsius. The temperature sensor is a system which needs a thermocouple or RTD (Resistance Temperature Detectors) to measure the temperature through an electrical signal. The analog signal is generated by the unit and is directly proportional to the temperature, if the voltage difference is enhanced.

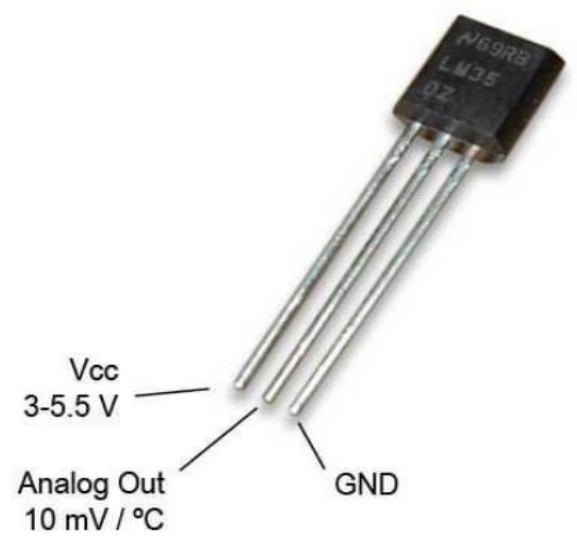

\section{Buzzer}

Figure 4. Temperature Sensor

Figure 5 shows the picture of a buzzer or beeper is a electronic, electromechanical or piezoelectrical (stretching system) audio signaling tool. Typical applications for buzzers include warning systems, clocks and user output confirmations such as a mouse click or keystroke. The buzzer consists of a metal case of two pins for control and field. Inside the portion there is a piezo dimension, comprising a central ceramic disc with a metal vibratory disk (often bronze). That results in the shaking of the surrounding disk. The tone you make. It is the noise you experience.

\section{Working:}

Piezo bells are straightforward gadgets that can create essential signals and tones. They work by utilizing a piezo precious stone, an uncommon material that changes shape when voltage is applied to it. In the event that the precious stone pushes against a stomach, similar to a little speaker cone, it can produce a weight wave which the human ear gets as sound.

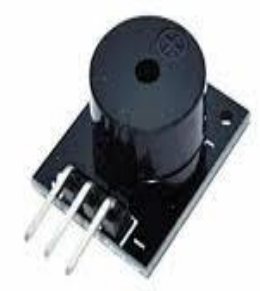

Figure 5. Buzzer

\section{E. BP Sensor}

The Blood Pressure Sensor shown in Figure 6, is a nonobtrusive sensor intended to quantify human pulse. It estimates systolic, diastolic and mean blood vessel pressure using the oscillometric technique.

The gadget comprises of two sections: a photoplethysmography (PPG) sensor and a flimsy shot power transducer. The PPG sensor estimates changes in blood volume by enlightening tissue and estimating changes in light assimilation, which can decide your pulse. 


\section{Working:}

A weight meter demonstrates the sleeve's weight. A little, handheld pneumatic machine blows up the pulse sleeve. After the sleeve has been expanded an air value is utilized to gradually discharge pneumatic force. A stethoscope will be used for checking in to the sounds in blood stream through the blood vessel as weight is released.

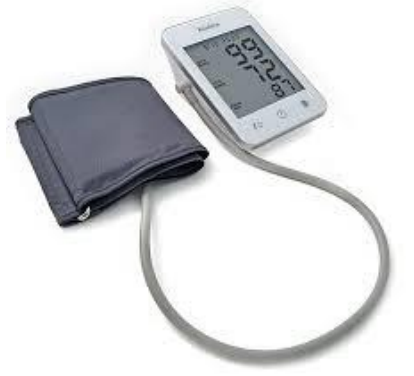

Figure 6. BP Sensor

\section{F) GPS Module (Global positioning System)}

This is used for distance identification-place determination. Navigation: go from one location to the next. Tracking: entity or motion control.

Geography: produces global charts.

Schedule: Specific day of the year.

The following three lines are composed of GPS, Which is shown in fig. 7 space segment (GPS satellites).

\section{Working:}

A network of about 30 satellites circling the Planet at an altitude of $20,000 \mathrm{~km}$ is the Global Positioning System (GPS). Such transmissions, which travel at the speed of light, are received by the GPS antenna, which determines how far a satellite is depending on how long it took the messages to arrive. There are many specific pieces of information on the data sent down from each satellite that enable the GPS receiver to determine their location and time accurately. An extremely accurate atomic clock is a significant piece of equipment on each GPS satellite. The period on the atomic clock, on the angular location and on arrival times of the satellite at various points in the atmosphere was transmitted back to the planet. In other terms, a timetable is given on any of the satellites observable, along with details on the position of the actual satellites (including other details parts) in the atmosphere. The GPS receiver now knows the distance to every satellite from this detail. When you can see at least 4 satellites from the GPS receiver antenna, your location and time can be correctly measured.

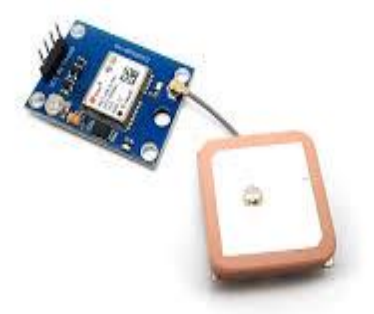

Figure 7. GPS Module

\section{G. GSM Module (Global system for Mobile communication)}

GSM is a handheld modem as shown in fig. 8 is used in our implementation. Abbreviation GSM stands for a Global system for mobile communication (GSM). In 1970, at Bell Labs, the concept of GSM was born. Mobile networking is commonly used in the world. Operating with $850 \mathrm{MHz}$, $900 \mathrm{MHz}, 1800 \mathrm{MHz}$, and $1900 \mathrm{MHz}$ frequency bands, GSM, Which is shown in Figure 8, is a free, wireless cell system that helps to transfer mobile voice and data services. GSM module is a chip or circuit which is used to link mobile devices or computers to a GSM or GPRS network. It consists of the GSM / GPRS Adapter, a SIM Card inserter, RS-232 Gateway, signal status Indicator, power supply and a communication provision for microphones and speakers. That module of GSM / GPRS is special and is distinguishable by its IMEI code.

\section{Working:}

Once a digital SMS order is obtained from a mobile phone, the SIM card mounted GSM modem sends the data to the MC through serial communication. The GSM modem gets the 'STOP' instruction to create the MC signal from which point of touch the ignition function is disabled when the system is going.

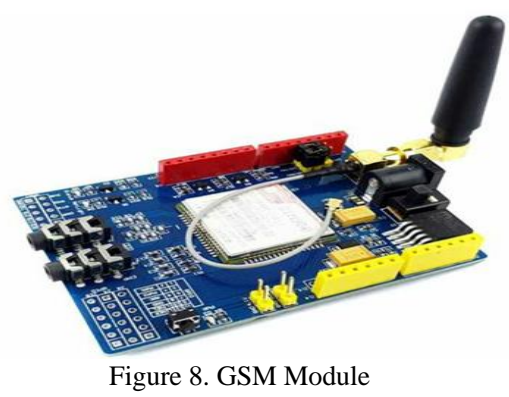

\section{H. LCD Display $16 \times 2$}

LCD (Liquid Crystal Display) is a kind of flat panel display that utilizes the key mode of activity for liquid crystals. LEDs are a wide and varied variety of use cases, typically used in smart telephones, TVs, computer displays and instrument panels, for customers and businesses. A flat-panel or other electro-modulated optical system is a Liquid Crystal Display that utilizes the light-modulating property in combination with polarizers of liquid crystals. Liquid crystals do not specifically emit light, but will produce images in color or monochrome using the backlight or reflector.

\section{Working:}

The application in fluid crystal displays operates by blocking light. In specific, two polarized glass parts (also called substratum) are created in the shape of an LCD that carries within them a fluid crystal substance. A lamp backlight creates lamp flowing into the first substratum.

An LCD, Which is shown in Figure 9 is a fluid crystal display module to generate a transparent image. A rather simple module is a 16-2 LCD panel, usually found in brickwork and circuits. The 16-point converts o to a 16-point view in 2 such lines per side. That character is depicted in this LCD in a matrix of 5 to 7 pixels. 


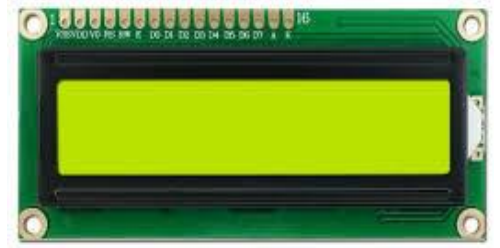

Figure 9. LCD Display

\section{METHODOLOGY}

A mobile monitoring system was developed and implemented for the transmission of a patient's temperature and HEART BEAT signal using short message service with low cost hardware equipment Via GSM module, the values obtained from sensors are interfaced with mobile Android.

Our project is Arduino based patient monitoring, a hardware device for tracking critically ill patients, various physiological parameters. The patients are tracked primarily because they are unbalanced in body system. The patient's problems can be monitored by continuous monitoring as they arise, and solutions taken before these problems get out of control. This project was developed and implemented with the goal to capture parameters.

The device is made of a motherboard microcontroller with all the peripherals. The voltage signal resulting from the output is fed to the external card where it is amplified to $0-5 \mathrm{~V}$ range and fed to the microcontroller card. The ADC in the microcontroller board transforms and feeds analog input signal into digital data into the computer.

\section{RESULTS AND DISCUSSION}

Results of our work carried out are shown below: Fig. 10 shows the interconnection of hardware components. It is a complete hardware system that read, transfer and trigger alerts.

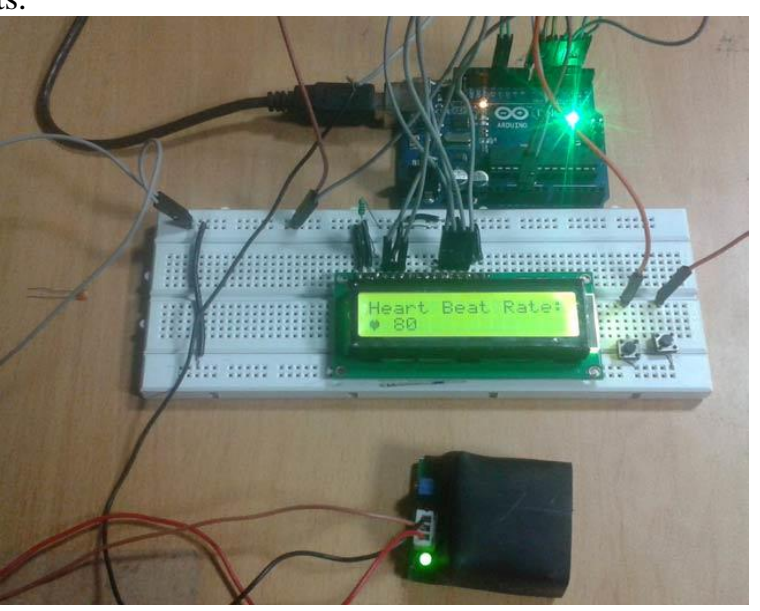

Figure 10. Heart Pulse Sensor

Fig. 11 shows the temperature of a patient body sensed by temperature sensor. Microcontroller is configured to trigger alert messages when the sensed temperature out lies the specified range. Normal temperature is set to $35^{\circ} \mathrm{C}$ to $39^{\circ} \mathrm{C}$. Output of blood pressure sensor is shown in Fig. 12.
Figures 13 and 14 shows connection and working of GPS and GSM components.

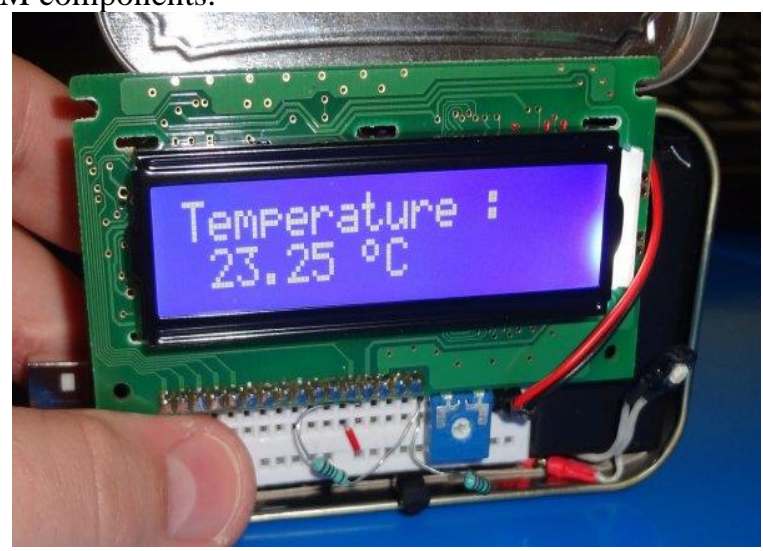

Figure 11. Temperature Sensor
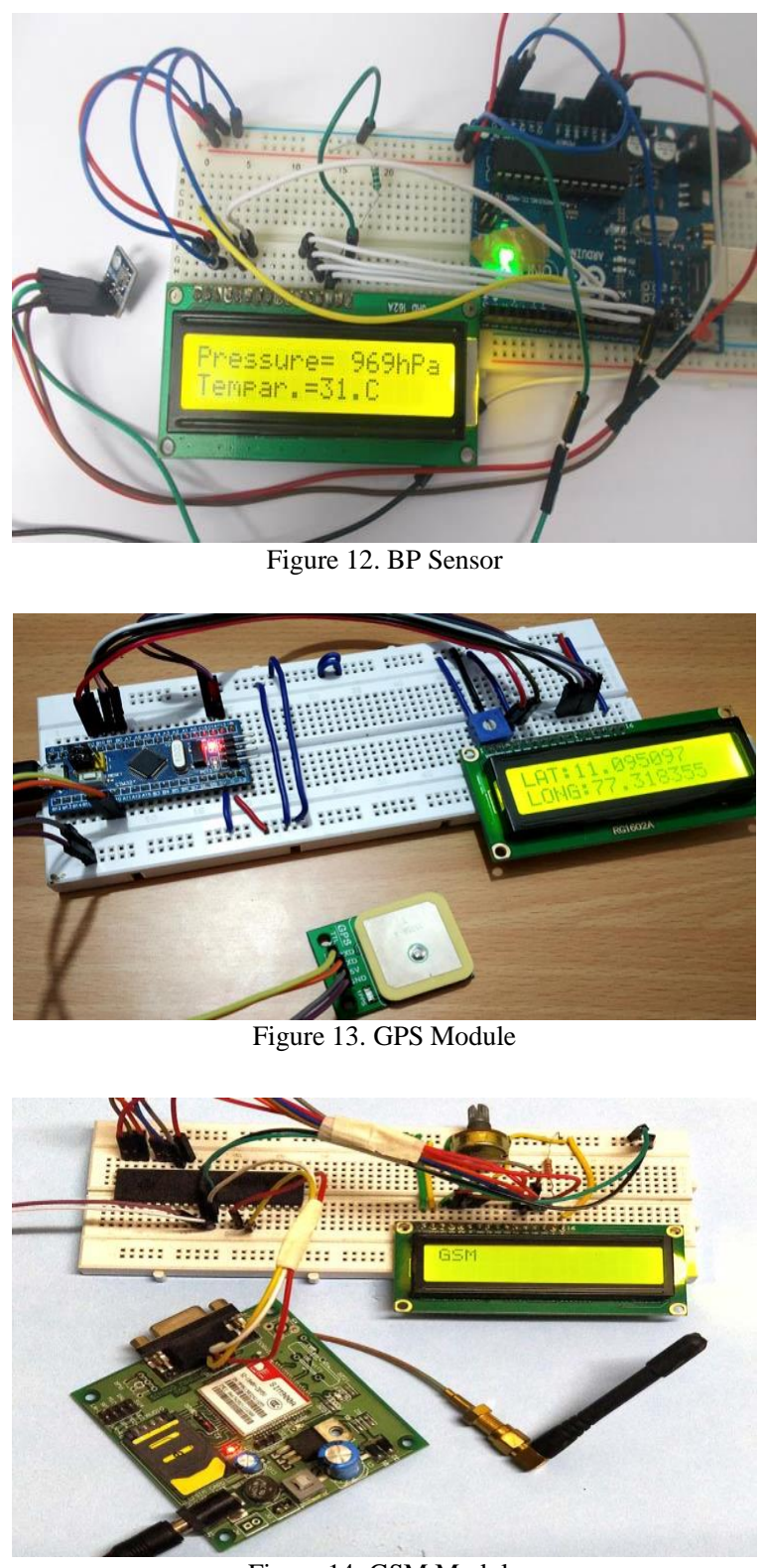

Figure 14. GSM Module 


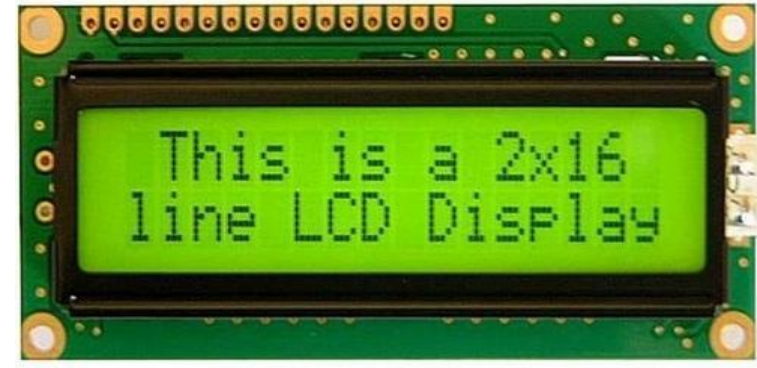

Figure 16. LCD Display

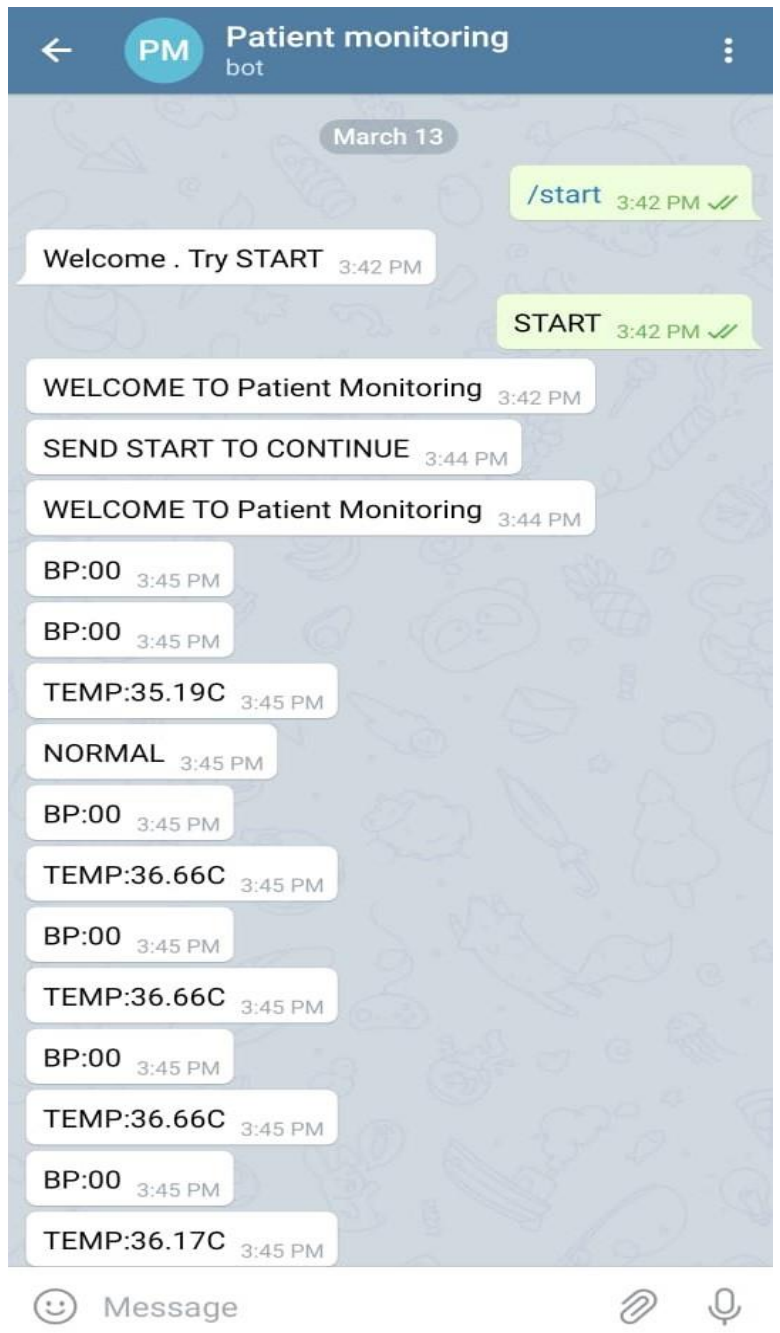

Figure 17. Android Application

\section{CONCLUSION}

Proposed system provides little effort, low power consumption and is extremely portable for patient health monitoring and can eliminate the need of expensive facilities. Medical practitioners can easily access the sick person's details anywhere, using the android application.

\section{REFERENCES}

[1] N. A. Yusuf, F. Y. Zulkifli and I. W. Mustika, "Development of Monitoring and Health Service Information System to Support Smart Health on Android Platform," 2018 4th International Conference on Nano Electronics Research and Education (ICNERE), Hamamatsu, Japan, 2018, pp. 1-6.

[2] Gu, J. Shen and Y. Chen, "Poster Abstract: Know You Better: a Smart Watch Based Health Monitoring System," 2019 IEEE/ACM International Conference on Connected Health: Applications, Systems and Engineering Technologies (CHASE), Arlington, VA, USA, 2019, pp. 7-8.

[3] O. Tartan and C. Ciflikli, "An Android Application for Geolocation Based Health Monitoring, Consultancy and Alarm System," 2018 IEEE 42nd Annual Computer Software and Applications Conference (COMPSAC), Tokyo, 2018, pp. 341-344.

[4] W. von Son et al., "Smart genetics for smarter health - an innovation proposal to improve wellness and health care in the cities of the future," 2017 International Smart Cities Conference (ISC2), Wuxi, 2017, pp. 1-4.

[5] Akshat et al., "A Smart Healthcare Monitoring System Using Smartphone Interface,"2018 4th International Conference on Devices, Circuits and Systems (ICDCS), Coimbatore, 2018, pp. 228231.

[6] A. O. Santos, G. S. de Jesus, G. A. Botelho and H. T. Macedo, "Smart health: Using fuzzy logic in the monitoring of health-related indicators," 2016 8th Euro American Conference on Telematics and Information Systems (EATIS), Cartagena, 2016, pp. 1-4.

[7] S. Ananth, P. Sathya and P. Madhan Mohan, "Smart Health Monitoring System through IOT," 2019 International Conference on Communication and Signal Processing (ICCSP), Chennai, India, 2019, pp. 0968-0970.

[8] N. Reddy, A. M. Marks, S. R. S. Prabaharan, S. Muthulakshmi, "Iot augmented health monitoring system", 2017 International Conference on Nextgen Electronic Technologies: Silicon to Software (ICNETS2), pp. 251-254, March 2017.

[9] $\mathrm{Yu}, \mathrm{W} . \mathrm{Xu}, \mathrm{N}$. Zhang and C. Yu, "Non-invasive smart health monitoring system based on optical fiber interferometers," 2017 16th International Conference on Optical Communications and Networks (ICOCN), Wuzhen, 2017, pp. 1-3. 